\title{
iDietScore $^{\mathrm{TM}}$ : Meal Recommender System for Athletes and Active Individuals
}

\author{
Norashikin Mustafa ${ }^{1}$ \\ ${ }^{1}$ Faculty of Health Sciences \\ Universiti Kebangsaan Malaysia, Kuala Lumpur, Malaysia \\ ${ }^{1}$ Department of Nutrition, Kulliyyah of Allied Health \\ Sciences, International Islamic University Malaysia \\ Kuantan, Malaysia \\ Abdul Hadi Abd Rahman ${ }^{2 *}$, Nor Samsiah Sani ${ }^{3}$ \\ Center for Artificial Intelligence Technology \\ Universiti Kebangsaan Malaysia, Bangi, Malaysia \\ Mohd Izham Mohamad ${ }^{4}$, Ahmad Zawawi Zakaria ${ }^{5}$ \\ National Sport Institute \\ Kuala Lumpur, Malaysia
}

\author{
Azimah Ahmad ${ }^{6}$ \\ National Defense University of Malaysia \\ Kuala Lumpur Malaysia \\ Noor Hafizah Yatiman ${ }^{7}$ \\ Ruzita Abd Talib ${ }^{8}$, Poh Bee Koon ${ }^{9}$ \\ Nutritional Science Program and Centre for Community \\ Health, Faculty of Health Sciences, Universiti Kebangsaan \\ Malaysia, Kuala Lumpur, Malaysia \\ Nik Shanita Safii ${ }^{10}$ \\ Dietetics Program and Centre for Community Health \\ Faculty of Health Sciences, Universiti Kebangsaan Malaysia \\ Kuala Lumpur, Malaysia
}

\begin{abstract}
Individualized meal planning is a nutrition counseling strategy that focuses on improving food behavior changes. In the sports setting, the number of experts who are sports dietitians or nutritionists (SD/SN) is small in number, and yet the demand for creating meal planning for a vast number of athletes often cannot be met. Although some food recommender system had been proposed to provide healthy menu planning for the general population, no similar solution focused on the athlete's needs. In this study, the iDietScore ${ }^{\mathrm{TM}}$ architecture was proposed to give athletes and active individuals virtual individualized meal planning based on their profile, includes energy and macronutrients requirement, sports category, age group, training cycles, training time and individual food preferences. Knowledge acquisition on the expert domain (the SN) was conducted prior to the system design through a semistructured interview to understand meal planning activities' workflow. The architecture comprises: (1) iDietScore ${ }^{\mathrm{TM}}$ web for SN/SD, (2) mobile application for athletes and active individuals and (3) expert system. SN/SD used the iDietScore ${ }^{\mathrm{TM}}$ web to develop a meal plan and initiate the compilation meal plan database for further use in the expert system. The user used iDietScore $^{\mathrm{TM}}$ mobile app to receive the virtual individualized meal plan. An inference-based expert system was applied in the current study to generate the meal plan recommendation and meal reconstruction for the user. Further research is necessary to evaluate the prototype's usability by the target user (athletes and active individuals).
\end{abstract}

Keywords-Expert system; meal planning; sports nutrition; inference engine; design and development

\section{INTRODUCTION}

Athletes need adequate energy and nutrition as fuel to sustain their long training hours and maintain their health [1]. Understanding an athlete's training periodization plan would give an idea or guideline for dietitians or nutritionists to match the nutrition strategies to support the training outcome [2]. Athlete training is divided into different cycles throughout the years and each of the cycles consists of different volume, frequencies and intensity of training sessions. Therefore, food for athletes should also change to meet different nutrition demands [3]. Several cross-sectional studies on athlete's dietary intake found that most of them did not meet their energy requirements during training and competition [4]-[7]. Besides, a systematic review identifies that most of the semiprofessional and professional team sports athletes exceed the needs of protein and fat during training and competition [6]. Inadequate nutrition intake not only occurred among adult or elite athletes but also affected young athletes. A systematic review by reference [8] identified that adolescent athletes (age 10-19 years old) did not adjust their nutrient intake based on their sport and intensity of training. Low energy intake among athletes may lead to several health consequences such as loss of muscle mass; menstrual dysfunction; loss of or failure to gain bone density; an increased risk of fatigue, injury, and illness; and a prolonged recovery process [1]. This condition may affect an athlete's carrier, performance and health. Therefore, action needs to be taken to improve athletes' dietary intake, especially during training and competition.

Meal planning is one of the nutrition counseling strategies that facilitate food behavior changes. Meal planning is a detailed meal plan listing precisely the type of food with the portion size to be eaten [9]. Moreover, meal planning is viewed as one technique to deliver nutrition knowledge in a more practical way [10]. According to four randomized control trial studies, preparing the meal plan was a helpful strategy in achieving health and food behavior changes among middle-aged adults [9]. An expert's knowledge of food composition, usually by a dietitian or a nutritionist, is needed to translate nutrition prescription into food choice and 
mealtime [11]. In the sports setting, the number of experts (sports dietitians or nutritionists) are small, and the demand for creating meal planning for a huge number of athletes often cannot be met. Moreover, traditional meal planning development using pen and paper is time-consuming.

Considering the fact that advanced technology may be used to assist people in improving health, the current study proposed an architecture design of iDietScoreTM, a system that provides virtual meal plans based on athlete's or active individual's profiles (include food preference) and expert's suggestion. The expert system provides a good platform for implementing technologies that may be identical or comparable to human experts. In this study, sports nutritionists who had domain knowledge of food options to produce the equivalent macronutrient meal planning for their athletes. Thus, athletes and active individuals able to receive meal planning at any time and location, especially when sports dietitians or nutritionist is not available. The present paper is organized as follows: Section 2 presents the related work; in Sections 3 and 4 the system design and development are described and finally, conclusions and future work are drawn in Section 5.

\section{RELATED WORKS}

Expert systems provide an excellent platform to implement applications that can be similar or near to human experts, such as diagnosing and assisting humans in decision-making, suggesting an alternative option to a problem and advising [12]. In recent years, the expert system's nutrition and balanced food domain has been discovered as a possible solution to direct the user to meet their personal nutrient needs [13]-[16]. Meal recommender or meal planning is considered as a multi-dimensional problem since it includes several decision variables with multiple constraints and objectives. In general, most of the study aims to develop a meal recommender based on nutrition recommendation [17] and recent studies include 'user's food preference [13][18][19]. Food preference is the key element of personalizing nutrition. Personalized nutrition has been defined in a number of ways, and this research describes it as an environment that empowers human autonomy to drive nutrition strategies that prevent, manage and treat diseases and improve health [20]. According to characteristics described by reference [21], personalized nutrition not only act as a disease preventive tools but it also empowers individuals to make a healthy choice according to their preferred foods and characteristics. Determine individual food preference is quite challenging as it depends on many factors such as culture, religion, knowledge and food availability [13], [22], [23]. A less palatable combination of food or unfamiliar food that suggests to the user might lead to non-adherence of the recommendation. Thus, the local food database is a crucial component to be included in the meal recommendation system.

In general, most studies with customized diet recommendations have few layers to process the information before the final recommendation. These layers include information gathering, user profile dataset, the intelligent system and the end-user interface [24]. The intelligence system usually focuses on receiving input from a user profile and produce output information of the recommended meal plan. The inference engine is one of the artificial intelligence techniques used in an expert system that applies a rule-based reasoning approach into the knowledge base in order to deduced recommendations [13], [25], [26]. The domain knowledge and rules were used to generate a recommendation. The benefit of rule-based reasoning is that it can solve the data shortage or cold start issue with machine learning and collaborative filtering approach. In addition, another advantage of the rule-based system is it has uniformity of knowledge format [13].

Knowledge acquisition is an essential process of the expert system, and it is quite challenging and time-consuming, but massive of information can be collected if the appropriate method is applied [27]. Knowledge can be acquired from different sources such as experts, book and documents [28][25]. The previous study had conducted knowledge acquisition in various techniques such as interview the domain expert, review the literature, document, guideline or related web site and observation [17], [25], [27], [29], [30]. A combination of interview and observation is recommended for acquired tacit and explicit knowledge [28]. Less research on the meal recommender for athletes is discussed. Reference [31] develops a suitable system for active individuals by providing a workout session and diet plans. However, nutrition rules for athletes did not include in this study. Moreover, reference [3] describes the development of a personalized food and nutrition ontology working with a rulebased knowledge framework to provide specific menus for the 'weightlifter's diary nutritional needs and personal preferences. However, this system was developed only for a single type of sport.

Therefore, there is still huge potential and opportunity to explore more on developing a system that specifically for athletes or active individuals. Sports dietitians or nutritionists would be the most suitable experts for knowledge acquisition purposed in the sports nutrition domain. Thus, the proposed approach in this study belongs to the rule-based approaches. Therefore, a rule-based approached was used in the current expert system to represent human expert knowledge.

\section{SYSTEM DESIGN}

\section{A. Knowledge Acquisition}

Knowledge acquisition was conducted to understand the process and workflow on how sports dietitians/ nutritionists (SD/SN) translate the athlete's information and profile into individualized meal plan. Knowledge acquisition was conducted among SD/SN currently working with national athletes in Malaysia through face to face interviews. The duration of an interview session was approximately 30 to 45 minutes. A semi-structured interview was conducted to give the participants room to answer the questions. Moreover, probes were used to explore the answers provided in-depth. The semi-structured interview guide were asked about the conditions that required meal planning and to describe the processes involved during the development of a meal plan. Probe questions such as "Can you explain further?" and "Following that, what else did you do?" were asked. The interviews were audio-recorded and transcribed verbatim. The 
transcripts that had been produced were then shared with the participants to check for the description's accuracy and adequacy. The validation of transcripts was important to make sure that the researcher's account truly reflected the true conversation [32] and to manage the issue of reliability or trustworthiness [33]. A thematic analysis was conducted and Atlas.ti 8 was used to support the labeling and retrieval of data that had been assigned a particular code [34]. This study adopted Braun and Clarke's (2006) step-by-step guidelines to create meaningful themes [35].

Table I presents six themes that emerged based on the interview and these themes were the general process that is involved in the development of meal planning for athletes practiced by SN. The sub-themes were the specific components that are important to be included in each theme or process in meal planning development.

\section{B. Architecture of iDietScore ${ }^{T M}$}

Based on the acquisition of expertise, high critical thinking and evidence-based practice relating to sports nutrition were required during the process of planning an athlete's meal plan. Meal planning is designed to include food options consistent with athletes' nutritional needs, training schedule and dietary preferences, as illustrated in Fig. 1. Expert systems provide a good platform for implementing technologies that may be identical or comparable to human experts, such as diagnosing, assisting people in decision-making, recommending solutions to a problem and offering advice [12]. The current expert system aims to provide a virtual meal plan based on nutrition needs, training plan, training time, and food preferences for athletes and active individuals. In order to achieve the aim, an architecture of iDietScore ${ }^{\mathrm{TM}}$ system (Fig. 2) was designed based on the workflow practiced by the $\mathrm{SN}$ in developing individualized meal planning. The interrelated structure of iDietScore $^{\mathrm{TM}}$ comprises: (1) iDietScore $^{\mathrm{TM}}$ web for sports dietitians or nutritionists, (2) mobile application for athletes and active individuals and (3) expert system.

The flow starts from the collection of meal plan database from SN using the iDietScore ${ }^{\mathrm{TM}}$ web. Next, using the iDietScore $^{\mathrm{TM}}$ mobile app, users must provide input on the profile page such as measurement of anthropometries, sports type, training cycle, training time, food preferences and food allergies. Based on the information, the system generates energy and nutrition requirement for the user. The expert system (ES), consisting of an inference engine (component 1), matches the user profile with a meal plan database by followed the meal plan rules that had been embedded in a knowledge base (component 2). ES proposes a meal plan that matches the user profile. Besides, ES also allows users to make changes in each food item in the meal plan by following meal reconstruction rules embedded in the knowledge base. All changes were recorded and save as a new meal plan. The descriptions of each architectural structure are addressed in the next sections that start with the iDietScoreTM web for sports dietitians or nutritionists and followed by mobile application for athletes and active individuals and expert systems.
TABLE I. SUMMARY OF THEMES AND SUBTHEMES

\begin{tabular}{|c|c|}
\hline $\begin{array}{l}\text { Themes (General } \\
\text { Process) }\end{array}$ & Sub-themes (specific process) \\
\hline $\begin{array}{l}\text { Collecting pertinent } \\
\text { data }\end{array}$ & $\begin{array}{l}\text { - Conducting body composition assessment } \\
\text { - Identify training periodization plan } \\
\text { - Identify training time } \\
\text { - Identify food and nutrition-related history }\end{array}$ \\
\hline $\begin{array}{l}\text { Analyzing the } \\
\text { collected data }\end{array}$ & $\begin{array}{l}\text { - Analyzing body composition } \\
\text { - Analyzing dietary intake }\end{array}$ \\
\hline $\begin{array}{l}\text { Determining } \\
\text { nutrition prescription }\end{array}$ & $\begin{array}{l}\text { - Calculating energy requirement } \\
\text { - Determining macronutrient distribution based on } \\
\text { g/kg body weight } \\
\text { - Using food exchange distribution table to distribute } \\
\text { macronutrient across the mealtimes }\end{array}$ \\
\hline $\begin{array}{l}\text { and determining } \\
\text { actions }\end{array}$ & $\begin{array}{l}\text { - Determining the use of supplements } \\
\text { - Emphasizing in gradual dietary changes strategy } \\
\text { - Setting achievable goals }\end{array}$ \\
\hline & $\begin{array}{l}\text { - Conducting one to one meeting between SNs and } \\
\text { athletes to discuss the meal plan }\end{array}$ \\
\hline $\begin{array}{l}\text { Recommending and } \\
\text { implementing action }\end{array}$ & $\begin{array}{l}\text { - Dietary education } \\
\text { - Adjusting and improvising current dietary intake } \\
\text { Determining mealtimes (main meal, pre \& post- } \\
\text { exercise meal) to match with training time }\end{array}$ \\
\hline Monitoring & $\begin{array}{l}\text { - Monitoring dietary intake } \\
\text { - Monitoring body composition }\end{array}$ \\
\hline
\end{tabular}

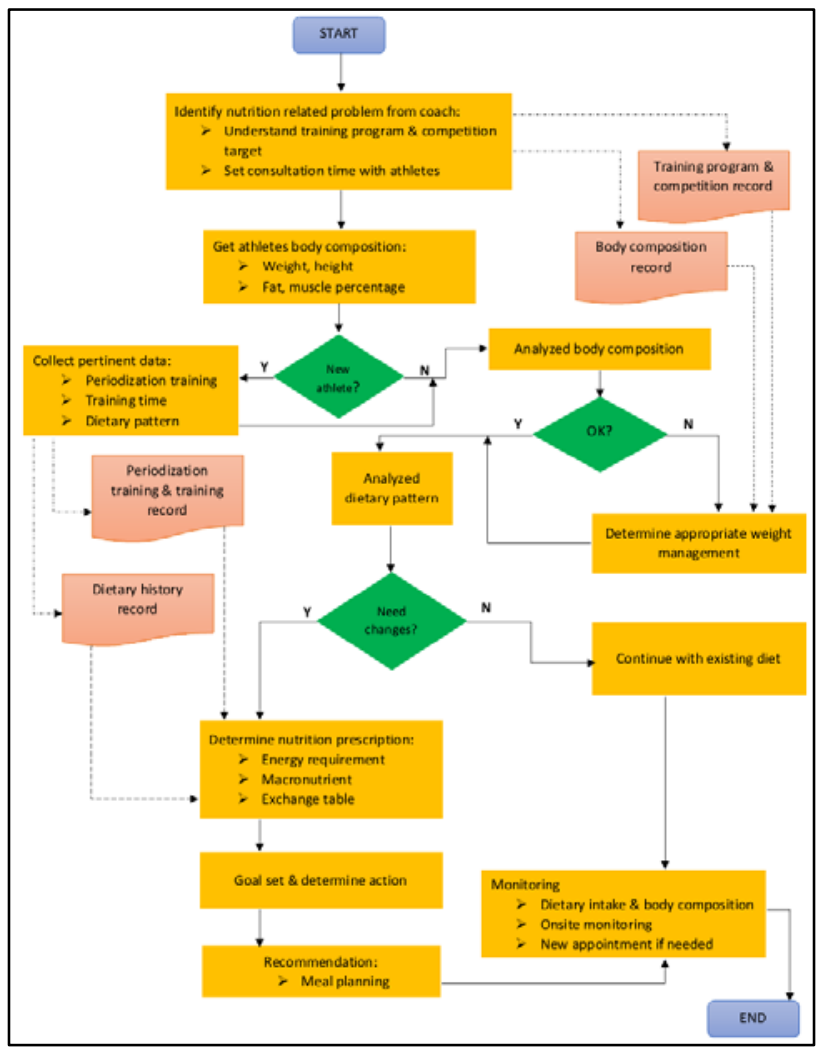

Fig. 1. The Workflow of Meal Planning Activities as based on Interviewing Sports Nutritionist in National Sports Institute, Malaysia. 


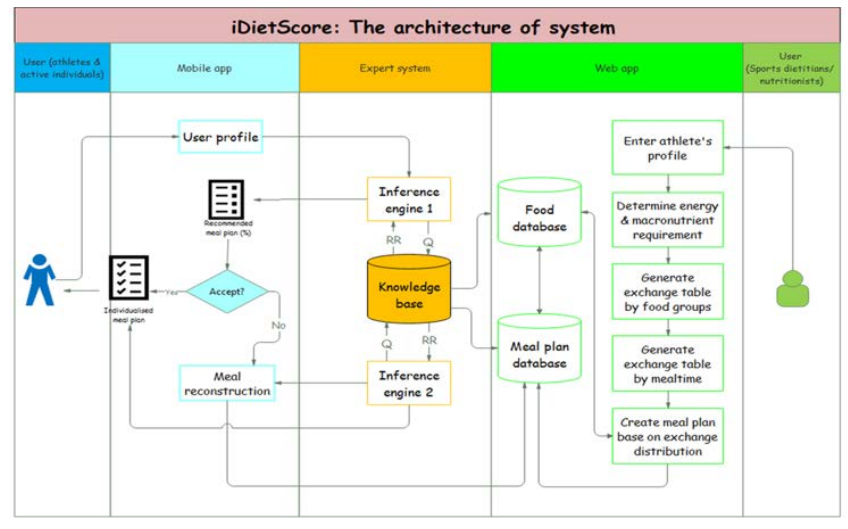

Fig. 2. A Design of iDietScore ${ }^{\mathrm{TM}}$.

\section{C. iDietScore ${ }^{T M}$ Web App for Sports Dietitians or Nutritionist $(S D / S N)$}

The traditional meal planning method with pen and paper takes time and lacks documentation. Thus, comprehensive meal planning sets cannot be compiled and reused for similar cases. iDietScore ${ }^{\mathrm{TM}}$ web has been developed to assist SD / SN plan a complete set of 1-day meals for athletes and active individuals that can be compiled into meal plan database. Moreover, the web also aims to initiate the compilation of meal plan database using a web application. The meal plan database is one of the important components in the development of the expert system for iDietScore ${ }^{\mathrm{TM}}$. It was design based on current practices by SNs (knowledge acquisition, Fig. 1), sports nutrition guidelines [36] [1] and food exchange list with macronutrient content by [37].

The web automatically calculates calories (in kcal) and macronutrient (in percentage, gram/day and food exchange distribution) requirements based on sports categories (such as endurance, intermittent/power strength, skill and active individual). The energy requirement was determined based on a formula calculation of two parameters which are basal metabolic rate (BMR) and physical activity level (PAL) (Energy requirement $=\mathrm{BMR} \times \mathrm{PAL})$ [38]. Thus, to come out with the requirement, input from SD/SN is still needed. SD needs to enter the user profile, verify the calculated calories recommendation, suggest suitable carbohydrate and protein intake, distribute the calculated food group exchange into mealtime and suggest appropriate food from the food database. The input from SD/SN to develop meal planning were illustrated in Fig. 3. The meal plan is saved as a whole set that is linked to the profile, such as total calories, sports categories, training time, season, food allergies and food preferences.

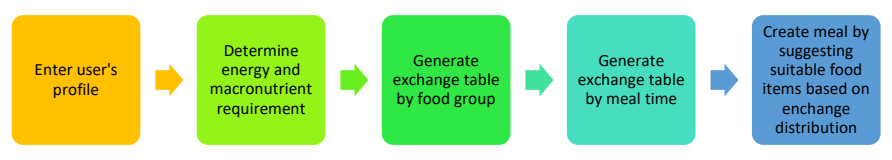

Fig. 3. A The required Input from SD/SN to Develop Meal Plan.

\section{D. iDietScore ${ }^{T M}$ Mobile App Features and Rule-Base Expert System}

The aim of iDietScore ${ }^{\mathrm{TM}}$ mobile app development is to assist the user who are athletes and active individuals to meet their calorie and nutrient requirements by suggesting them with the individual meal plan. The individual meal plan is based on their current nutrient needs, sports type, training time, training cycle, food allergies and food preferences. In addition, the user can also change the food that is suggested in the meal plan but within the control nutrient values. The rulebased expert system (ES) was type of ES that being develop in current study to generate the recommendation of meal plan and meal reconstruction for the user. The rule-based ES comprises of three main components which are user interface, inference engine and knowledge base. All information about user profiling (such as age, gender, weight, height, type of sports, training time, food allergies and food preferences) was collected at the user interface. Those information were essential for energy calculation and macronutrient (carbohydrate and protein) recommendation. The calculation was similar as described in iDietScore ${ }^{\mathrm{TM}}$ web.

The next component is the knowledge base that contains the specialized knowledge of the domain problem. The current study includes rules related to individualized meal plan together with rules for meal reconstruction. All the rules were acquired from the experts (sports nutritionists), sports nutrition position statement and nutrition guideline represented in the declarative form of "if.... then..." rule. This study implements forward chaining as the inference engine follow the chain of conditions or rules to deduce the outcome. This study has two inference engines to differentiate between expert inference engine for meal plan (E1) and inference engine for meal reconstruction (E2). The $1^{\text {st }}$ rule involves meal planning recommendations. Referring to the architecture (Fig. 2), upon receiving the profile input from the user, inference engine 1 (E1) will infer with meal plan recommendation (at least one meal plan) together with the score of accuracy. The accuracy of the meal plan suggested by the ES is seen in the percentage. The more rules that are followed, the greater the quality of that meal plan. The indicator will offer users a view of how reliable the meal plans to meet their nutrient needs. There are five rules for meal planning suggestions that are included in the knowledge-based. The flow chart in Fig. 4 shows how the rules (label with the alphabetic start from A until E ) are being applied in the inference engine 1 (E1) to produce a meal plan suggestion to a specific user.

A higher score (50\%) will be given as the meal plan meets the energy requirement (Rule A). Next, a score of 30\% was given as the meal plan meets the sports categories' rule. Sports categories resemble the macronutrients distribution thus, meeting this rule will be receiving a more accurate meal plan. A score of $10 \%$ was given as the meal plan meet the rule that related to training time. Training time is related to mealtime distribution. Next, another $5 \%$ was given as the meal plan meet each of the rules related to food allergies and food preferences. The total of $100 \%$ would refer to the most accurate meal plan or meet all rules for meal plan. 


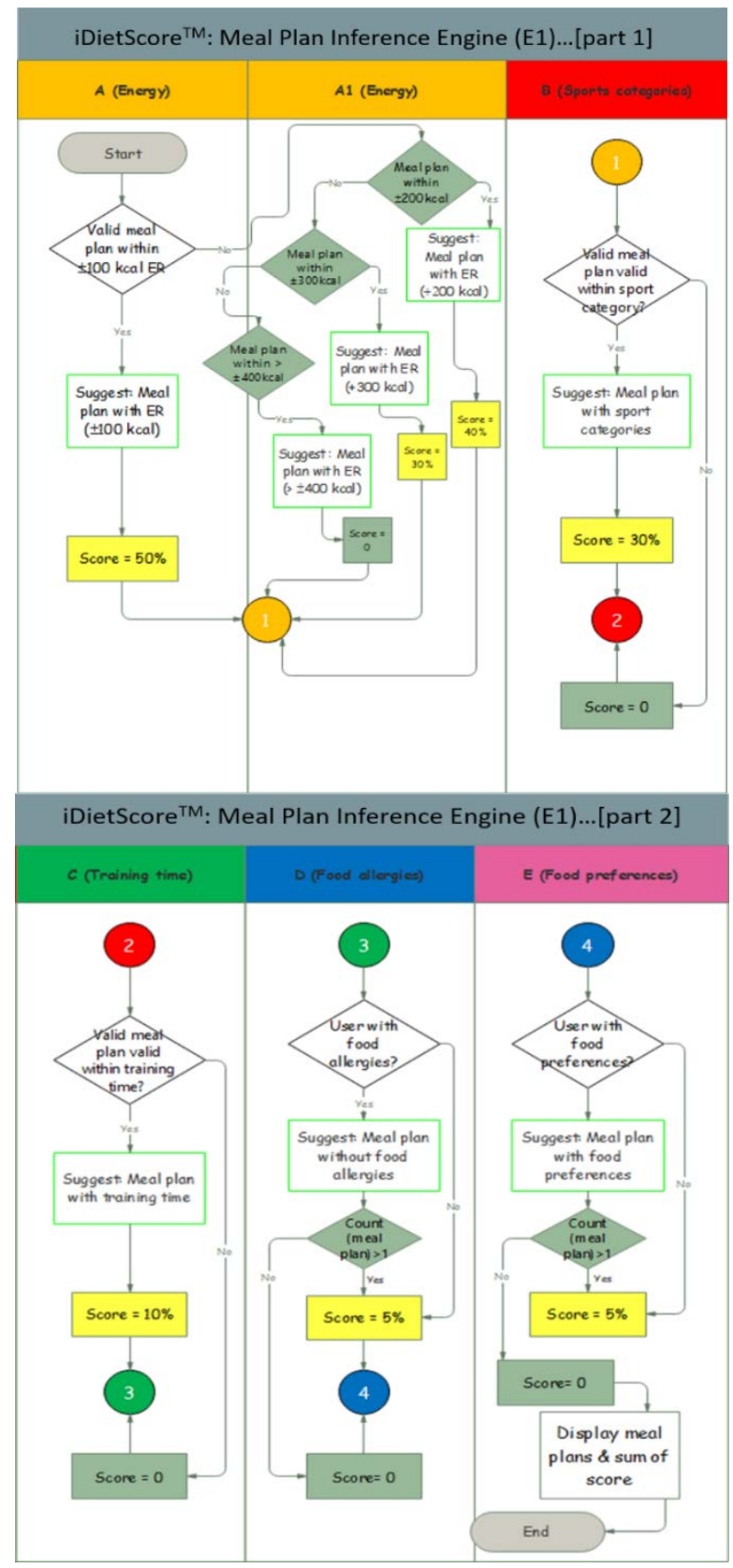

Fig. 4. A the Flow of Meal Plan Inference Engine (E1).

Based on the system architecture, as the user received the meal plan list, they must choose one that they preferred. Next, as they agreed with all the food that is suggested in the meal plan, they must confirm it, and that particular meal plan is called an individualized meal plan. However, they have an option to change any specific food that they disagreed within the meal plan. Thus, inference engine 2 (E2) will infer a suitable food for changes based on meal reconstruction rules. Three rules must be met for meal reconstruction that included in the knowledge base. The rules are labeled with alphabetic and the details are described in Table II. Rule A related to the food group meanwhile rule B is about the calories of current food. In rule $\mathrm{B}$, the calorie allowance is depending on the food group. A new food group (Food group A - Food group D) was created to classify them based on calorie allowance. The aim is to make sure the food list will not be exceeding or lacking any macronutrients and at the same time able to provide varieties of food choices to the user. The concept is to include a user's preference in planning on their meal plan within the control environment. The process flow (Fig. 5) shows how the rules are being applied in the inference engine 2 (E2) for food changes in meal reconstruction. The meal reconstruction aims to include user participation in developing their meal plan by allowing them to choose food that they preferred while being controlled by specific rules. The applied rules assure the calories and macronutrient is within the recommendation.

\section{E. Database}

The availability of the local food and beverages database is a critical component that can contribute to mobile app user satisfaction, especially in the food record feature. This study modified the food database established by [39] to develop a sports nutrition mobile app. The current study added another 2000 common Malaysian multi-ingredient menus in the database selected from the National Sports Council of Malaysia cafeteria and sports school dining hall. The food items were label into 32 food groups, six food types,13 food exchange groups and seven mealtimes (breakfast, lunch, dinner, snacks, pre-exercise meal, during and post-exercise meal). The food database contains the information on energy (kcal), house-hold measurement unit, eight nutrient contents (in gram) of over 3000 food items that are commonly eaten and prepared in Malaysian.

Meal plan databases were compiled using iDietScore ${ }^{\mathrm{TM}}$ web app. SD/SN from ISN was voluntary use the web and develop a meal plan. In order to make sure the meal plan database can cover most of the athlete's profile, SD/SN create a meal planning based on calories (of 1500, 2500, 3500 and $4500 \mathrm{kcal}$ ), sports categories (of endurance, intermittent/power strength, skill and active individuals), season ( of "on season" and "off-season") and training time (morning training, evening training and morning and evening training). There was 120 meal plan database were collected and used in the expert system. The meal plan number is expected to be increased as the web is released and used by more SD/SN.

TABLE II. The THREE RULES OF MEAL ReCONSTRUCtion

\begin{tabular}{|l|l|l|l|}
\hline & Rules description & If ..then.. & Example \\
\hline A & Food group & $\begin{array}{l}\text { IF current food group is } \\
\text { A } \\
\text { THEN list out food that } \\
\text { within food group A }\end{array}$ & $\begin{array}{l}\text { If user want to } \\
\text { change apple } \\
\text { (from food group } \\
\text { named fruits), the } \\
\text { food list must } \\
\text { come from fruits. }\end{array}$ \\
\hline B & $\begin{array}{l}\text { Calorie: } \pm 100 \\
\text { (total calorie)* }\end{array}$ & $\begin{array}{l}\text { IF current calorie is B } \\
\text { THEN list out food that } \\
\text { within calories } \pm 10 \text { of B } \\
\text { not exceed total 100 } \\
\text { kcal/day }\end{array}$ & $\begin{array}{l}\text { If apple calories is } \\
\text { 75 kcal, the list of } \\
\text { fruits must within } \\
\pm 10 \text { of 75kcal }\end{array}$ \\
\hline C & $\begin{array}{l}\text { Mealtime } \\
\text { (Breakfast, Snack, } \\
\text { Lunch, Dinner, } \\
\text { pre-exercise, } \\
\text { during and post- } \\
\text { exercise) }\end{array}$ & $\begin{array}{l}\text { IF current mealtime is C } \\
\text { THEN list out food that } \\
\text { tag with mealtime C }\end{array}$ & $\begin{array}{l}\text { If user want to } \\
\text { change food for } \\
\text { breakfast, the list } \\
\text { of new food must } \\
\text { also suitable for } \\
\text { breakfast. }\end{array}$ \\
\hline
\end{tabular}




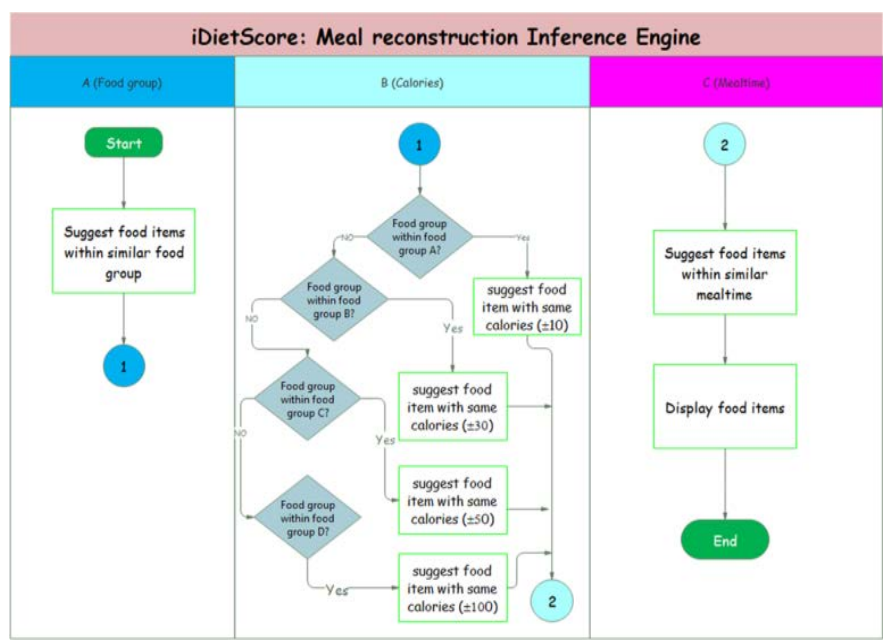

Fig. 5. The Flow of Meal Reconstruction Inference Engine (IE 2).

\section{F. Development and Expert Evaluation of a Prototype}

Agile methodologies, an iterative process of development, were applied in this study. Agile development promotes adaptive planning, evolutionary development, early delivery, continuous improvement, and encourages rapid and flexible response to change [40]. This approach makes the developers more aware of the changing nature of the project and at the same time, also lets them give feedback to the design process [41]. The agile process will force all the members to collaborate and coordinate among team members during the development process [41]. Thus, the integration of agile methodologies is believed to improve the communication between two different fields of knowledge, such as health and technology. Fig. 6 shows the design of important features in iDietScore $^{\mathrm{TM}}$ web for SD/SN. Fig. 7 shows the design of essential features in iDietScore ${ }^{\mathrm{TM}}$ mobile app for athletes and active individualized.

The expert evaluation was conducted upon the completion of both prototypes. Eight expert panels (in sports nutrition area) were involved. They need to evaluate the relevancy of features content, appropriateness, easiness, and accuracy of the language used and the attractiveness and appropriateness of the graphic user interface (GUI) based on the four-point scale. Scale one indicates not relevant/appropriate/easy/ accurate/attractive. Scale two means the item needs revision, scale three is relevant but needs minor revision and scale four is very relevant/appropriate/ easy/accurate/attractive. All experts (8/8) agreed that all the features content was relevant to the target population (55\% of experts rated the features as very relevant and the remaining rated the features as relevant but need minor revision). Moreover, $75 \%$ (6/8) of experts rated the language used in web and mobile app were appropriate, easy to understand and accurate. Lastly, $62.5 \%$ (5/8) viewed that the GUI was appropriate and attractive.
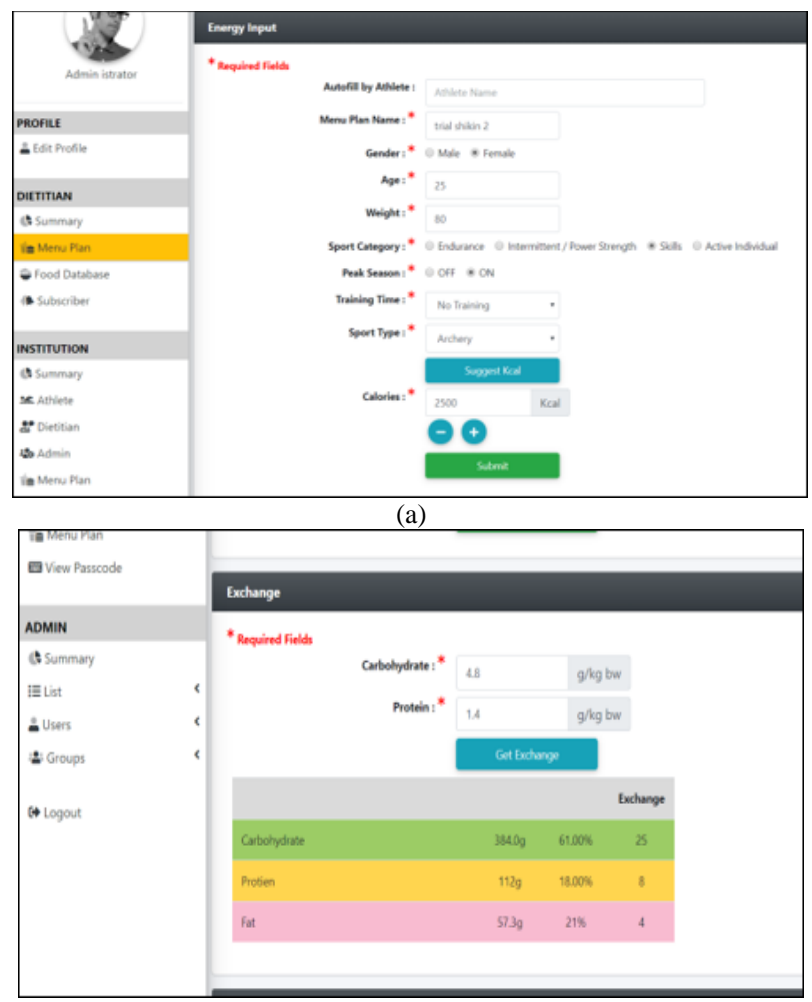

(b)

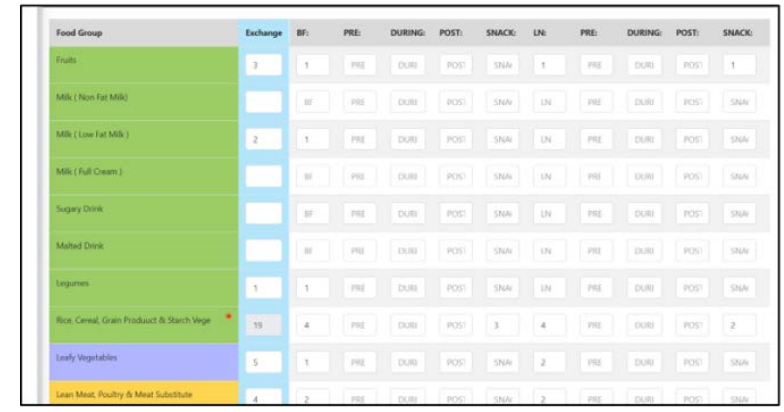

(c)

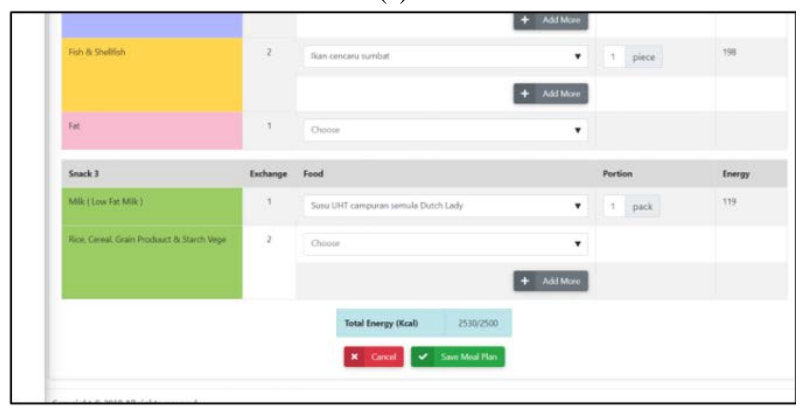

(d)

Fig. 6. Snapshot of Important Features in iDietScore ${ }^{\mathrm{TM}}$ Web for Sports Dietitians or Nutritionist: (a) Enter user's Profile; (b)Determine Energy and Macronutrient (c) Generate Exchange Table by Food Groups and Mealtime; (d) Create Meal Plan Meal by suggesting Suitable Food Items based on Exchange Distribution. 

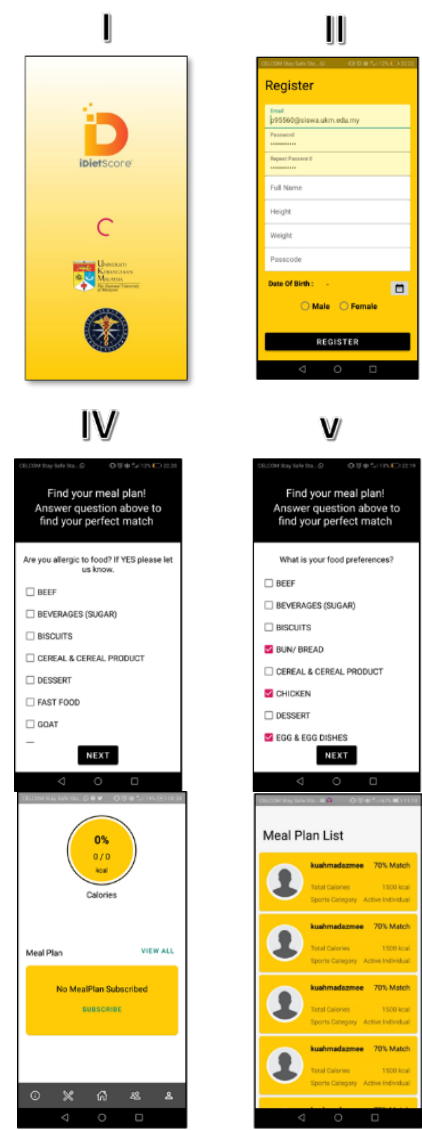

VII

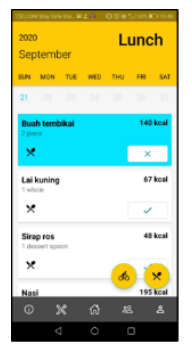

X

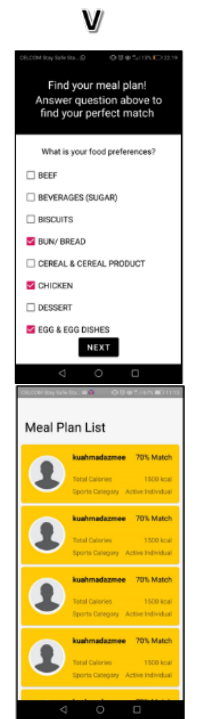

VIII

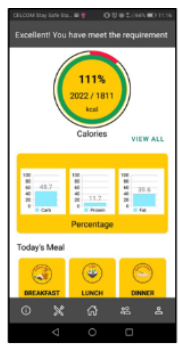

XI
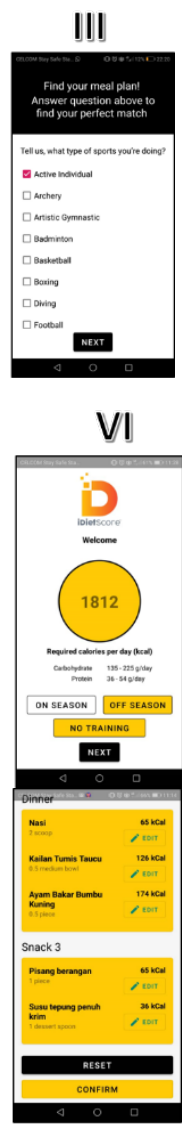

$\mathrm{IX}$

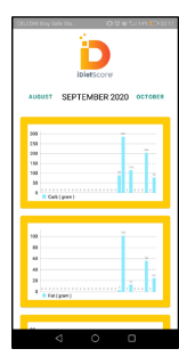

XII
Fig. 7. Snapshot of Main Features in iDietScore ${ }^{\mathrm{TM}}$ Mobile App for Athletes and Active Individuals: (I-VI) Athletes Profile that Include Height, Weight, Sports Type, Food Allergic, Food Preferences, Seasons and Training Time;

(VII) Subscribe Meal Plan; (VIII) List of Meal Plan that Suits Athletes

Profile; (IX) Meal Reconstruction; (X) Food Log; (XI) Home Page with

Current Energy and Macronutrient Intake; (XII) Analysis for 1 month.

\section{CONCLUSION AND FUtURE WORK}

In conclusion, the design of iDietScore ${ }^{\mathrm{TM}}$ was developed to provide a virtual meal plan based on nutrition need, training plan, training cycle (the season) and food preferences for athletes and active individuals. The rule-base expert system consisting of an inference engine matches a user profile with a meal plan database through meal plan rules integrated into the knowledge base. Moreover, the meal reconstruction rule set in the iDietScoreTM expert system's knowledge base allowed the user to change any suggested food items in the original meal plan into a food item that they preferred without altering their macronutrient needs. Users' participation in creating meal plans is another key element of the individualized meal plan, ultimately creating a sense of control and motivation to help individuals maintain short- and long-term adherence.

The future study would be the usability test (formative and summative testing) among target users. The formative testing aims to reveal several usability related problem that faces by the target user. These problems can be fixed or solve before final development is deployed. The think-aloud method was commonly used captured user experience of using the prototype. Meanwhile, the summative focus on the efficacy of the final system development and to obtain definitive evidence of usability on the final product. In addition, two experts in sports nutrition will independently evaluate the suitability of meal planning recommended by the inference engine based on 30 case studies that mimic the user's profile. The interrater agreement can be measured to make sure the rating obtained are not distinctive results of one rater's subjective judgment.

\section{ACKNOWLEDGMENT}

The authors want to thank the National Sports Institute Malaysia and Universiti Kebangsaan Malaysia for funding and supporting this research under grant code: NN-2018-093.

\section{REFERENCES}

[1] D. T. Thomas, K. A. Erdman, and L. M. Burke, "Position of the Academy of Nutrition and Dietetics, Dietitians of Canada, and the American College of Sports Medicine: Nutrition and Athletic Performance," J. Acad. Nutr. Diet., vol. 116, no. 3, pp. 501-528, 2016.

[2] T. Stellingwerff, J. P. Morton, and L. M. Burke, "A framework for periodized nutrition for athletics," Int. J. Sport Nutr. Exerc. Metab., vol. 29, no. 2, pp. 141-151, 2019.

[3] P. Tumnark, J. P. Vilas-boas, P. Cardoso, and J. Cabral, "OntologyBased Personalized Dietary Recommendation for Weightlifting Ontology-Based Personalized Dietary Recommendation for Weightlifting," Int. Work. Comput. Sci. Sport., no. December, 2014.

[4] J. Dwyer, A. Eisenberg, K. Prelack, W. O. Song, K. Sonneville, and P. Ziegler, "Eating attitudes and food intakes of elite adolescent female figure skaters : a cross sectional study," pp. 1-7, 2012.

[5] A. R. Jagim, H. Zabriskie, B. Currier, P. S. Harty, R. Stecker, and C. M. Kerksick, "Nutrient Status and perceptions of energy and macronutrient intake in a Group of Collegiate Female Lacrosse Athletes," vol. 7, pp. 17, 2019.

[6] S. L. Jenner, G. L. Buckley, R. Belski, B. L. Devlin, and A. K. Forsyth, "Team Sport Athletes Do Not Meet Sport Nutrition Recommendations — A Systematic Literature Review," no. 3, pp. 1-16, 2019.

[7] S. L. Jenner et al., "Dietary intake of professional Australian football athletes surrounding body composition assessment," J. Int. Soc. Sports Nutr., vol. 15, no. 1, pp. 1-8, 2018.

[8] M. Noll, C. R. De Mendonça, L. P. De Souza Rosa, and E. A. Silveira, "Determinants of eating patterns and nutrient intake among adolescent athletes: A systematic review," Nutr. J., vol. 16, no. 1, pp. 1-11, 2017.

[9] J. M. Spahn et al., "State of the Evidence Regarding Behavior Change Theories and Strategies in Nutrition Counseling to Facilitate Health and Food Behavior Change," J. Am. Diet. Assoc., vol. 110, no. 6, pp. 879891, 2010.

[10] C. J. Boushey, A. M. Coulston, C. L. Rock, and E. Monsen, Nutrition in the prevention and treatment of disease. Academic Press, 2001.

[11] J. G. Mirtschin et al., "Organization of Dietary Control for NutritionTraining Intervention Involving Periodized Carbohydrate Availability and Ketogenic Low-Carbohydrate High-Fat Diet," Int. J. Sport Nutr. Exerc. Metab., vol. 28, no. 5, pp. 480-489, Sep. 2018.

[12] W. K. Chen, The electrical engineering handbook. United States of America: Elsevier Academic Press, 2004. 
[13] S. Alian, J. Li, and V. Pandey, "A Personalized Recommendation System to Support Diabetes Self-Management for American Indians," Inst. Electr. Electron. Eng., vol. 6, pp. 73041-73051, 2018.

[14] M. Burgermaster et al., "A new approach to integrating patientgenerated data with expert knowledge for personalized goal setting: A pilot study," Int. J. Med. Inform., vol. 139, no. February, p. 104158, 2020.

[15] C. H. Chen, M. Karvela, M. Sohbati, T. Shinawatra, and C. Toumazou, "PERSON - Personalized Expert Recommendation System for Optimized Nutrition," IEEE Trans. Biomed. Circuits Syst., vol. 12, no. 1, pp. 151-160, 2018.

[16] I. Marinchev and G. Agre, "An expert system for healthful and dietary nutrition," ACM Int. Conf. Proceeding Ser., vol. 1164, no. June, pp. 229-236, 2016.

[17] S. A. Noah et al., "DietPal: A Web-based dietary menu-generating and management system," J. Med. Internet Res., vol. 6, no. 1, pp. 32-48, 2004.

[18] G. Jaswal, A. Kaul, and R. Nath, "Knuckle print biometrics and fusion schemes - Overview, challenges, and solutions," ACM Comput. Surv., vol. 49, no. 2, 2016

[19] H. Jung and K. Chung, "Knowledge-based dietary nutrition recommendation for obese management," Inf. Technol. Manag., vol. 17, no. 1, pp. 29-42, 2016.

[20] C. L. Bush et al., "Toward the Definition of Personalized Nutrition: A Proposal by The American Nutrition Association," J. Am. Coll. Nutr., vol. 39, no. 1, pp. 5-15, 2020.

[21] A. T. Limon-miro, V. Lopez-teros, and H. Astiazaran-garcia, "Dynamic Macronutrient Meal-Equivalent Menu Method: Towards Individual Nutrition Intervention Programs," methods Protoc., vol. 2, no. 3, p. 78, 2019.

[22] A. Kale and N. Auti, "Automated menu planning algorithm for children: food recommendation by dietary management system using ID3 for Indian food database," in Procedia Computer Science, 2015, vol. 50, pp. 197-202.

[23] L. Yang et al., "Yum-Me: A personalized nutrient-based meal recommender system," ACM Trans. Inf. Syst., vol. 36, no. 1, 2017.

[24] R. Yera Toledo, A. A. Alzahrani, and L. Martinez, "A food recommender system considering nutritional information and user preferences," IEEE Access, vol. 7, pp. 96695-96711, 2019.

[25] I. M. Ahmed, M. Alfonse, and M. A. A. M. Salem, "Daily Meal Planner Expert System for Diabetics Type-2," E-Leader Prague, no. 2007, 2015.

[26] M. V Gupta, P. Bhattacharjee, and N. Kotian, "DANES: Diet and Nutrition Expert System for Meal Management and Nutrition Counseling," Int. J. Recent Innov. Trends Comput. Commun. , vol. 5, no. 12, pp. 204-208, 2017.
[27] B. A. Al-dhuhli, "Developing a Nutrition and Diet Expert System Prototype," no. JUNE 2013, pp. 1368-1375, 2013.

[28] A. H. Mohammad and N. A. M. Al Saiyd, "A Framework For Knowledge Acquisition," Int. J. Comput. Sci. Netw. Secur., vol. 10, no. May, pp. 42-50, 2010.

[29] V. Espín, M. V. Hurtado, and M. Noguera, "Nutrition for Elder Care: A nutritional semantic recommender system for the elderly,” Expert Syst., vol. 33, no. 2, pp. 201-210, 2016.

[30] D. Ribeiro, J. Machado, J. Ribeiro, M. J. M. Vasconcelos, E. F. Vieira, and A. Correia de Barros, "SousChef: Mobile Meal Recommender System for Older Adults,” no. Ict4awe, pp. 36-45, 2017.

[31] F. Mata, M. Torres-Ruiz, R. Zagal, G. Guzman, M. Moreno-Ibarra, and R. Quintero, "A cross-domain framework for designing healthcare mobile applications mining social networks to generate recommendations of training and nutrition planning," Telemat. Informatics, vol. 35, no. 4, pp. 837-853, 2018.

[32] A. Pilnick and J. A. Swift, "Qualitative research in nutrition and dietetics: Assessing quality," J. Hum. Nutr. Diet., vol. 24, no. 3, pp. 209-214, 2011.

[33] C. Anderson, "Presenting and Evaluating Qualitative Research: Strengths and Limitations of Qualitative Research," Am. J. Pharm. Educ., vol. 74, no. 8, pp. 1-7, 2010.

[34] A. Draper and J. A. Swift, "Qualitative research in nutrition and dietetics: Data collection issues," J. Hum. Nutr. Diet., vol. 24, no. 1, pp. 3-12, 2011.

[35] V. Braun and V. Clarke, "Using thematic analysis in psychology," Qual. Res. Psychol., vol. 3, no. 2, pp. 77-101, Jan. 2006.

[36] C. M. Kerksick et al., "ISSN exercise \& sports nutrition review update: Research \& recommendations," J. Int. Soc. Sports Nutr., vol. 15, no. 1, pp. 1-57, 2018.

[37] S. Shahar, N. S. Safii, Z. Manaf, and H. Haron, Atlas Makanan: Saiz Pertukaran \& Porsi, 3rd editio. Kuala lumpur: MDC Publishers Sdn Bhd, 2015.

[38] N. N. R. Rodriguez, N. Di Marco, S. Langley, and N. M. DiMarco, "American College of Sports Medicine, American Dietetic Association, and Dietitians of Canada joint position statement: Nutrition and athletic performance.," Med. Sci. Sports Exerc., vol. 41, no. 3, pp. 709-731, 2009.

[39] S. Nik Shanita et al., "DietScoreTM: Sports Nutrition-based Mobile Application for Athletes and Active Individuals," in IFMBE Proceedings, vol. 58, 2017, pp. 1-5.

[40] C. Quesada-lópez et al., "Design , Development and Validation of a Mobile Application for Goal Setting and Self-Monitoring of Dietary Behaviors," no. 2004, 2009.

[41] E. A. Altameem, "Impact of Agile Methodology on Software Development," vol. 8, no. 2, pp. 9-14, 2015. 